In Crescendo, 2019; 10(1): 89-114

Fecha de recepción: 03 de noviembre del 2018

Fecha de aceptación: 25 de marzo del 2019

\title{
INTERDISCIPLINARIEDAD EN EL APRENDIZAJE DEL SISTEMA DE ENSEÑANZA MODULAR POR OBJETOS DE TRANSFORMACIÓN EN LA UNIVERSIDAD AUTÓNOMA DE SINALOA
}

\author{
INTERDISCIPLINARITY IN THE LEARNING OF THE MODULAR \\ TEACHING SYSTEM BY OBJECTS OF TRANSFORMATION \\ AT THE UNIVERSIDAD AUTÓNOMA DE SINALOA
}

\section{Patricia Carmina Inzunza-Mejía Ana María López-Carmonal}

\section{RESUMEN}

El objetivo de este artículo es analizar y comprender los desafíos que teórica, empírica y conceptualmente implica desarrollar y aplicar la metodología de la investigación en el aprendizaje de una o varias disciplinas. Es una investigación con enfoque cualitativo bajo el método inductivo de investigación-acción-participativa. La población participante es de alumnos que cursan el Sistema Modular por Objetos de Transformación (SEMOT) de la Facultad de Ciencias Económicas, Administrativas y Tecnológicas de la Universidad Autónoma de Sinaloa en México. Las técnicas de análisis e interpretación de datos fueron la observación participante, el cuestionario y la entrevista semiestructurada. Los hallazgos revelan que la debilidad en el aprendizaje y conocimiento de asignaturas nodales, como metodología de la investigación, comprensión de textos científicos y resolución de problemas, representa una de las principales limitaciones y dificultades para desarrollar contenidos teóricos interdisciplinares y modelos teóricos metodológicos, restringiendo el buen desarrollo del SEMOT.

PALABRAS CLAVE: Interdisciplinariedad, Sistema Modular, objeto de transformación, transferencia del conocimiento, vida colegiada.

1 Universidad Autónoma de Sinaloa. 


\begin{abstract}
The objective of this article is to analyze and understand the challenges that theoretically, empirically and conceptually involve developing and applying the methodology of research in the learning of one or more disciplines. It is a research with a qualitative approach under the inductive method of action-participatory research. The participating population is of students who study the Modular System for Objects of Transformation (SEMOT) of the Faculty of Economics, Administrative and Technological Sciences of the Autonomous University of Sinaloa in Mexico. The techniques of data analysis and interpretation were participant observation, questionnaire and semi-structured interview. The findings reveal that the weakness in the learning and knowledge of nodal subjects, such as research methodology, understanding of scientific texts and problem solving, represents one of the main limitations and difficulties to develop interdisciplinary theoretical contents and methodological theoretical models, restricting the good development of the SEMOT.
\end{abstract}

KEY WORDS: Interdisciplinarity, Modular System, Object of Transformation, Transfer of knowledge, Collegiate Life.

\title{
INTRODUCCIÓN
}

Formar en competencias investigativas a los estudiantes de nivel superior es cada vez más importante para su desarrollo profesional. En la Facultad de Ciencias Económicas, Administrativas y Tecnológicas (FCEAT) de la Universidad Autónoma de Sinaloa (UAS), desde hace 36 años se aplica el sistema de enseñanza modular por objetos de transformación (SEMOT) con el que los estudiantes vinculan una o varias disciplinas con problemas del contexto socioeconómico regional y nacional.

La formación en el SEMOT se complementa con asignaturas como metodología de la investigación, comprensión y producción de textos científicos, análisis y resolución de problemas, y creatividad e innovación. Su desarrollo se perfecciona con la incorporación de cursos de diferentes denominaciones disciplinares, orientados a fortalecer las competencias específicas de investigación para que pueda ejecutar actividades que vinculen el conocimiento con los problemas de su entorno profesional.

En la mayoría de las escuelas y facultades de la Universidad Autónoma de Sinaloa y otras Instituciones de Educación Superior (IES) de México, se evita el significado de la actividad investigativa. La mayoría de las veces, el propósito de promover la formación bajo un sistema de enseñanza orientada a la investigación se 
deja en segundo término, evitando discutir su pertinencia e importancia con una o más disciplinas, lo que limita la interdisciplinariedad e interinstitucionalidad.

Para desarrollar la interdisciplina en las IES, es fundamental promover la formación en investigación, además de analizar y discutir la enseñanza de la ciencia y sus articulaciones disciplinares. Para Aiello-Sindoni (2009:143), "las decisiones de los profesores e investigadores adoptan la formación en investigación, lleva implícita asociar determinadas posturas y concepciones sobre qué es y sobre cómo se analiza y se produce el conocimiento científico".

El SEMOT se focaliza en la formación para la investigación mediante el método investigación-acción y se visibiliza en proyectos de investigación y planes de intervención según sea el contexto, campo de estudio o disciplina en la que se aplique.

En este estudio se plantea: ¿Qué desafíos enfrentan los estudiantes de FCEAT-UAS a partir del 6to semestre para desarrollar el proceso de aprendizaje a través del Sistema de Enseñanza Modular por Objetos de Transformación?, ¿Qué beneficios ofrece el desarrollo del SEMOT?

\section{METODOLOGÍA}

Esta investigación tiene un enfoque cualitativo de tipo descriptivo, que, en función de la formulación de la pregunta de investigación, se desarrolla con base a la contrastación entre la revisión del estado del arte y la fundamentación empírica para un razonamiento deductivo, lo que permite generar categorías y relacionar variables que, en conjunto, favorecen la comprensión del objeto de estudio.

La unidad de análisis son 3 Licenciaturas que se imparten en la Facultad de Ciencias Económicas, Administrativas y Tecnológicas de la Universidad Autónoma de Sinaloa, en México. Estas Licenciaturas son la Licenciatura en Negocios Internacionales, la Licenciatura en Negocios Agro-tecnológicos y la Licenciatura en Contaduría Pública Fiscal, mientras que el objeto de estudio es el SEMOT y su impacto en la interdisciplinariedad.

Los sujetos de estudio se corresponden a 652 estudiantes que estudian estas Licenciaturas, distribuidos en cuatro semestres (del sexto al noveno), En estos semestres se imparte el SEMOT. Los 652 estudiantes representarían la población analizada, que conforme al $95 \%$ de nivel de confianza y un margen de error del $5 \%$ se identifica una muestra de 242 estudiantes. 
Los participantes del estudio fueron 72 alumnos de la Licenciatura en Negocios Internacionales, 76 alumnos de la Lic. en Negocios Agro-tecnológicos y 94 alumnos de la Licenciatura de Contaduría Pública Fiscal.

A los estudiantes se aplicó un cuestionario con 47 ítems acerca de los principales problemas que se les presentaban para desarrollar el SEMOT y los desafíos que tuvieron para la identificación y descripción de conceptos y bases teóricas vinculadas a los temas de la unidad de aprendizaje modular que en cada semestre desarrollan, operacionalización de variables y competencias relacionadas al proceso de investigación, así como la percepción que tienen acerca del SEMOT y su aplicación.

Como informantes clave, también participaron 10 profesores investigadores de tiempo completo que han impartido el SEMOT y colaborado en el desarrollo de los trabajos de investigación modular. Se aplicó entrevista estructurada acerca de las principales dificultades y desafíos que los estudiantes presentan respecto al desarrollo disciplinar y metodológico del SEMOT y qué condiciones de enseñanza son necesarias para mitigar estas dificultades. También se aplicó la observación participante al estar inmersos en el objeto de estudio y en permanente interacción con los sujetos de investigación.

\section{REVISIÓN DE LITERATURA}

Pese a la fragmentación histórica del conocimiento científico en ciencias naturales y sociales, se ha reconocido que comparten desafíos y riesgos; surgiendo de ahí, el estado actual de un enfoque interdisciplinario. Para Martínez et al. (2018), la interdisciplina es el andamiaje que vincula distintas disciplinas, estimulando investigaciones adicionales

Bibri (2018), considera que la investigación académica en el campo de las ciencias sociales se basa en el entendimiento de que los avances en el conocimiento subyacente requieren la búsqueda de respuestas a preguntas multifacéticas y multifactoriales. Esto solo puede resolverse desde el punto de vista de la interdisciplinariedad o la transdisciplinariedad mediante el establecimiento de un marco que relacione analíticamente varias disciplinas; lo que, proporciona una visión de integridad y complementariedad de un conjunto de teorías y disciplinas que se fusionan para ponerse en práctica al resolver un problema social.

Tal como lo expresara Cannizzaro (2013) al explorar la utilidad de la interdisciplinariedad como método de investigación y al proponerlo como concepto de información con características diversificadas, complementarias e integrado- 
ras, que al igual que Luckie (2013) sugiere que los futuros científicos deberán utilizar la investigación interdisciplinaria para comprender sistemas complejos, comunicar estas ideas a sus compañeros y desarrollar hipótesis comprobables.

En tal sentido, Luckie (2013), sugiere que el conocimiento compartido podría ser la técnica más efectiva para ayudar a los estudiantes introductorios en sistemas de investigación, en la comprensión de la ciencia en términos integrados; es decir, desde las conexiones de las disciplinas con el mundo real en una clase pequeña, tal cual se realiza con el Sistema de Enseñanza por Objetos de Transformación.

Sin embargo, MacLeod (2018), cuestiona las dificultades que enfrenta la interdisciplinariedad y presenta algunas consecuencias de la especificidad de dominio en la práctica interdisciplinaria, al igual que Piso et al. (2016), al referirse a los desafíos complejos de las ciencias sociales ante la desincorporación a otras ciencias. En este sentido, se entiende que la investigación interdisciplinaria se ha concentrado en la expectativa de que la interacción disciplinaria mejore a través de estrategias de las instituciones de educación y desde cambios de los sistemas de revisión por pares, pese a que los obstáculos institucionales y el diseño de políticas educativas dificultan la interdisciplina.

Piso et al. (2016) destaca que la capacidad integradora debe mantenerse en las investigaciones interdisciplinares, en virtud de aprovechar el complemento o compensación de otros enfoques disciplinares a la disciplina propia o especificidad de dominio. En este sentido, se demuestra que los estudios sociales de la ciencia pueden poner de relieve cómo los investigadores interdisciplinarios deberán trabajar sus estudios desde una concepción integrada.

$\mathrm{Al}$ respecto, se puede inferir que con la interacción de disciplinas se alcanzaría la capacidad integradora. Mientras que el diseño de estrategias y políticas educativas enfocadas a una educación científica podría favorecer la práctica científica y el desarrollo de la investigación, propiciando condiciones cognitivas particulares y mitigando las barreras metodológicas y conceptuales que se enfrentan los investigadores al trabajar en los límites de una disciplina.

En este sentido, MacLeod (2018:697) considera que "las lecciones aprendidas de la ciencia cognitiva y los estudios antropológicos de los laboratorios de sociología de la ciencia sugieren que las prácticas científicas pueden ser muy específicas del dominio". Sin embargo, aún no se comprende completamente como la especificidad de dominio de una disciplina restringe la investigación interdiscipli- 
naria, lo que representa un área de oportunidad para las ciencias sociales y la practica científica de la interdisciplina.

Coincidiendo con los estudios de Aranda et al. (2014), en la perspectiva integradora de las disciplinas, Abramo, et al. (2017), analizan la tendencia de diversificación de las actividades de investigación que algunos científicos a nivel mundial propician. Descubren que la colocación disciplinaria de la producción científica de científicos individuales es baja, y destacan que las investigaciones empíricas tienen menor grado de diversificación en matemáticas, mientras que existe una mayor diversificación e intensidad en investigadores de las ciencias sociales, lo que favorece la interdisciplina. Asimismo, los estudios de Camy et al. (2017), sostienen que el desarrollo de una cultura común y una curiosidad compartida respecto a otras disciplinas distintas de la propia, son factores clave para el desarrollo de la investigación interdisciplinaria sostenible.

Con base al análisis de la cuestión de la interdisciplinariedad en las actividades de investigación se concluye que en el SEMOT es fundamental un encuadre teórico acerca de estudios de varias disciplinas que aborden un hecho de la sociedad, principalmente en nuestra Facultad que forma profesionistas en ciencias económicas y administrativas con asignaturas y módulos compuestos por la disciplina esencialmente económica, administrativa, jurídica y contable. De ahí que la interdisciplina irá enriqueciendo los nuevos elementos teóricos, según sea el comportamiento de los hechos empíricos; es decir, el estado del arte teórico de lo que se esté indagando, será utilizada de acuerdo con los hechos en la realidad para desarrollar nuevos fundamentos teóricos, conceptos y relaciones categóricas de las manifestaciones reales observadas.

Además, de acuerdo con Ruskanen et al. (2018), con el enfoque interdisciplinario aplicado en un sistema de enseñanza como el SEMOT, se da la oportunidad de realizar estudios exploratorios, descriptivos y explicativos del aprendizaje, en donde el estudiante adquiere habilidades transferibles.

Dada la tendencia hacia la enseñanza interdisciplinaria y el diseño instruccional mediante el SEMOT, con el enfoque interdisciplinario también se diseñan los aportes curriculares desde una disciplina o varias disciplinas relacionadas, generando una ventaja absoluta en la creación de actividades que integran disciplinas, incorporan una diversidad de perspectivas científicas y consideran las opciones de diseño curricular instructivo y por competencias, garantizando la conectividad y uso de las tecnologías de la información, (Stubbs, et al., 2018). 
El desarrollo de competencias investigativas permite realizar investigación interdisciplinaria para plantear problemas de la realidad contextual que demanda solución y respuesta desde diferentes campos de estudio (Frank, et al. 2017). De ahí que aprender haciendo, implica apropiarse del conocimiento en la aplicación práctica de la solución del problema.

Aprender a través del SEMOT implica vivenciar el proceso de investigación científica. Así lo precisan Sullivan y Puntambekar (2019), al momento que los maestros inducen a los estudiantes al uso del método científico, utilizando un diálogo interdisciplinario para generar condiciones orientadas al uso de diversos recursos científicos como parte del proceso de hacer ciencia.

Para Liu et al. (2018), el aprendizaje se genera desde estrategias de intervención que de manera introspectiva y autónoma el estudiante interioriza para disponerse a aprender y transferir conocimientos, pero desde bases tecnológicas, haciendo uso de los sistemas de computación científicos. Desde esta visión, la parte introspectiva y autónoma de disposición y asimilación, es lo que genera el aprendizaje, que al aplicarse mediante el uso de las tecnologías de la información modernas se genera como refuerzo a la trasferencia de conocimiento, propio del SEMOT.

Esta característica de refuerzo en el aprendizaje se enfoca en el aprendizaje por transferencia de campos de actuación disciplinar o metodológico, con dominio diferente pero complementario, lo que permite un dominio cruzado entre la disciplina y el procedimiento científico; es decir, "los flujos de trabajo de diferentes dominios (por ejemplo, científicos o de negocios) tienen similitudes y diferencias entre sí que permiten que algunas soluciones desarrolladas en un dominio pueden ser fácilmente aplicables al otro", Koohi-Var y Zahedi (2018:2).

En este sentido, la enseñanza científica propia del SEMOT, comprende diversas prácticas metodológicas que dan sustento teórico y empírico al conocimiento práctico y a la investigación científica. Esto de acuerdo con Durham et al. (2018), y Auerbach y Andrew (2018), impacta en el crecimiento y desarrollo de la enseñanza y la productividad científica, basada en instrumentos y técnicas de investigación para descubrir las soluciones a problemas determinados, generando así el aprendizaje activo; sin embargo, la motivación y las habilidades socioemocionales del estudiante juega un papel fundamental.

Es claro que el conocimiento práctico generado desde las estrategias de la enseñanza y las conductas del aprendizaje donde la motivación del estudiante es 
fundamental, se genera conocimiento relacionado con la gestión científica fuera del aula. Esta condición está presente en la práctica del SEMOT, desde el momento que un estudiante sale a la práctica profesional a resolver un problema relacionado con la disciplina que está estudiando en un curso modular. De ahí que para Vezzani et al. (2018:665), "las concepciones del aprendizaje están relacionadas con factores personales y contextuales", donde el género, nivel de estudios y área académica de desempeño profesional son determinantes para el aprendizaje de los estudiantes universitarios.

Otro de los desafíos que enfrentan los estudiantes en el desarrollo del SEMOT, es el proceso de análisis y retención del conocimiento. En este sentido, el enfoque propuesto por Wikström et al. (2018:1510), destaca que "el proceso de retención de conocimiento, incluida la gestión y búsqueda de información científica, la codificación de ese conocimiento y la internalización del conocimiento, son aspectos clave de la gestión del aprendizaje", donde no solo la motivación; sino también la edad, son determinantes para generar el aprendizaje y la retención de este. Es claro que la retención del conocimiento implica una vinculación permanente de los sujetos con el objeto de estudio, además implica que la motivación sea un factor determinante para superar los desafíos en el proceso de aprendizaje y desarrollo de la investigación científica.

Además, ha quedado demostrada que la experiencia previa propicia una actitud proactiva y de apertura a la investigación científica favorece su desarrollo. Un estudio en Turquía realizado por Ünver et al. (2018), demuestra que quienes han participado previamente en actividades científicas mantiene una actitud positiva hacia la investigación, que aquellos que no han realizado investigación o desconocen el proceso de la metodología científica.

Pareciera contradictorio que el proceso de evaluación se desarrolle para generar aprendizaje, pero no lo es, porque son actividades complementarias.

Stark et al. (2018), sugiere que las instituciones de educación superior, los departamentos y programas educativos deben declarar el esquema de evaluación tanto del proceso de aprendizaje del estudiante como de los programas y cursos. El proceso de evaluación en todas sus dimensiones representaría una oportunidad para desarrollar competencias y habilidades de los estudiantes relacionados con el conocimiento científico y la investigación, para lo cual es fundamentar incluir a los estudiantes en el proceso de diseño, actualización, desarrollo, administración e interpretación de las evaluaciones. 
La evaluación de la competencia científica de los estudiantes es un proceso que permite analizar el desempeño productivo. Kruit et al. (2018:415) expresa que "la capacidad de los estudiantes para realizar una investigación científica está significativamente relacionada con la capacidad cognitiva general" que deviene del aprendizaje activo y el conocimiento aplicado; sin embargo, la competencia científica no se relaciona con la aplicación de exámenes escritos, desarrollo de informes metacognitivos, pruebas orales y revisión de avances de investigación.

También se puede evaluar el aprendizaje desde un marco genérico de razonamiento científico y argumentativo. En el análisis de Dorfner et al. (2018), como producto de la evaluación del aprendizaje generado desde la investigación científica, se mostró un rendimiento potencial en los estudiantes, por lo que consideran que el marco genérico de razonamiento científico y argumentativo con el que desarrollan actividades epistémicas es una herramienta metodológica valiosa para enseñar ciencias y fomentar el aprendizaje de los estudiantes.

Asimismo, el estudio de Dem et al. (2018), evalúa las motivaciones personales y los resultados de aprendizaje desde la investigación científica, donde ser parte de la investigación científica resultó ser uno de los tres factores determinantes para que los estudiantes estén motivados en hacer investigación científica.

Otro aspecto en la evaluación del aprendizaje mediante la investigación científica es la experiencia de enseñanza / aprendizaje multifacético que destaca Chen (2018) al precisar que con el desarrollo de la investigación científica, los estudiantes de Licenciatura son introducidos a diversos temas de investigación a través de la búsqueda, gestión, lectura, discusión de la literatura científica que conduce a la presentación y escritura de síntesis y reportes de investigación para difundir sus conocimientos generales y habilidades prácticas en la investigación científica.

En el SEMOT se desarrolla este proceso descrito por Chen (2018) y se presenta para su evaluación en un coloquio de investigación modular, lo que ayuda a los estudiantes aplicar la evaluación intelectual de la disciplina y sus habilidades de escritura científica.

\section{DISCUSIÓN Y CONTEXTO}

La construcción de escenarios y condiciones de investigación son fundamentales para el desarrollo del SEMOT, dado que permite desarrollar investigación científica responsable desde el aprendizaje activo, aplicado, reflexivo y cola- 
borativo. De esta manera, Betten et al. (2018), consideran que la construcción de tales escenarios contribuye al desarrollo de una investigación científica socialmente responsable para generar aprendizaje proactivo desde experiencias previas, inclusión, análisis, reflexión y capacidad de respuesta.

$\mathrm{Al}$ respecto, para alcanzar el aprendizaje desde la investigación transferible, el estudiante debe retener, comprender y aplicar el conocimiento. Para que esto suceda, Ruskanen et al. (2018) consideran que los estudiantes dedicados a realizar investigación científica necesitan habilidades transferibles, como las habilidades de aprendizaje y las habilidades de comunicación oral y escrita, a la luz del conocimiento que se transfiere y se aplica como se hace en el SEMOT.

\section{EXTENSIONES DEL ANÁLISIS}

La investigación acerca de la enseñanza-aprendizaje mediante la integración de la disciplina, acentúa el alto valor de la complementariedad del contenido, el aprendizaje, la investigación y la interdisciplina (Belardo, et al., 2017). De ahí que, los investigadores de las ciencias económicas y administrativas utilizan un escenario social, económico y empresarial como plataforma para realizar la investigación.

En diversas disciplinas científicas, han aumentado los métodos para buscar, gestionar, registrar, almacenar y analizar información científica. Para Huppenkothen et al. (2018), el uso de las herramientas científicas como bases de datos científicas, software para el procesamiento de datos, análisis y producción de artículos científicos, demandan capacitación en nuevos lenguajes y marcos de programación digital, así como la inmersión en nuevos modos de interacción colaborativa e intercambio entre disciplinas.

Para Kimmons y Veletsianos (2018:492-494), "los problemas técnicos, metodológicos, profesionales y éticos que surgen al involucrarse en métodos científicos y manejo de minería de datos públicos incluyen la necesidad de experiencia y rigor multifacéticos, preguntas de investigación enfocadas y determinación de significado, y consideraciones contextuales y de rendimiento de los datos públicos".

No obstante, la influencia de la investigación científica en el aprendizaje genera apertura mental, dado que permite el uso de diversas estrategias de aprendizaje que conducen a un aprendizaje reflexivo, realizable desde una comunicación didáctica que visibiliza la interlocución del profesor y el estudiante. Betten et al. (2018), reflexiona que los escenarios para una investigación socialmente responsable deben orientarse a la construcción del conocimiento y para generarlo se 
requiere generar un proceso reflexivo desde un escenario preciso y un contexto organizado para realizar la investigación.

\section{Contextualización del SEMOT dentro de los planes de estudios en FCEAT}

La Facultad de Ciencias Económicas, Administrativas y Tecnológicas (FCEAT) de la Universidad Autónoma de Sinaloa, se localiza en la Ciudad de Guamúchil Sinaloa, México. Desde 1982 se implementa el sistema modular que se concibe en sus inicios como aquel modelo educativo que "se caracteriza por ser crítico, global y activo. Enfrenta al alumno desde el inicio de su carrera a problemas concretos de su práctica profesional y por medio de la investigación científica de cada uno de ellos, con la consulta bibliográfica y la investigación de campo se le va ofreciendo todos los contenidos disciplinares, tanto teóricos como instrumentales que lo capaciten para ser un profesionista eficiente".

Tabla 1

MAPA CURRICULAR DE LA OFERTA EDUCATIVA DE FCEAT 2017-2018

\begin{tabular}{|c|l|l|l|}
\hline Semestre & \multicolumn{1}{|c|}{$\begin{array}{c}\text { Lic. Negocios } \\
\text { internacionales }\end{array}$} & \multicolumn{1}{c|}{$\begin{array}{c}\text { Lic. Negocios } \\
\text { agrotecnologicos }\end{array}$} & \multicolumn{1}{c|}{$\begin{array}{c}\text { Lic. Contaduría } \\
\text { pública fiscal }\end{array}$} \\
\hline Sexto & $\begin{array}{l}\text { Economía internacional } \\
\text { Economía digital y } \\
\text { cadenas de valor }\end{array}$ & $\begin{array}{l}\text { Uso potencial del suelo } \\
\text { Aprovechamiento de } \\
\text { recursos naturales e } \\
\text { industrializables }\end{array}$ & $\begin{array}{l}\text { Derecho corporativo } \\
\text { Finanzas }\end{array}$ \\
\hline Séptimo & $\begin{array}{l}\text { Estrategias negocios } \\
\text { internacionales } \\
\text { Negocios electrónicos }\end{array}$ & $\begin{array}{l}\text { Infraestructura para la } \\
\text { producción } \\
\text { Certificación de } \\
\text { productos y procesos }\end{array}$ & $\begin{array}{l}\text { Contribuciones de } \\
\text { personas físicas y } \\
\text { personas morales } \\
\text { Presupuestos }\end{array}$ \\
\hline Octavo & $\begin{array}{l}\text { Mercadotecnia } \\
\text { internacional } \\
\text { Comercio internacional }\end{array}$ & $\begin{array}{l}\text { Aprovechamiento de } \\
\text { material biotecnológico } \\
\text { Formulación de } \\
\text { proyectos }\end{array}$ & $\begin{array}{l}\text { Auditoría I } \\
\text { Contribuciones al } \\
\text { comercio exterior }\end{array}$ \\
\hline Noveno & $\begin{array}{l}\text { Derecho y gestión de } \\
\text { aduanas } \\
\text { Finanzas } \\
\text { internacionales }\end{array}$ & $\begin{array}{l}\text { Nutrición y profilaxis, } \\
\text { manejo ecológico de } \\
\text { plagas y malezas }\end{array}$ & $\begin{array}{l}\text { Auditoria II } \\
\text { Derecho procesal fiscal }\end{array}$ \\
\hline
\end{tabular}

Fuente: Elaboración propia con información Plan Curricular 2002 de Facultad de Ciencias Económicas, Administrativas y Tecnológicas (2017) de la Universidad Autónoma de Sinaloa. 
El SEMOT, se desarrolla en la etapa investigativa profesionalizarte del Plan de Estudios, a partir del sexto semestre de las Licenciaturas en Negocios Internacionales, Negocios Agro-tecnológicos y Contaduría Pública Fiscal que se ofrecen en FCEAT UAS. Constituye un sistema de formación para la investigación. Los créditos horarios en el semestre equivalen a 270 horas efectivas, divididas en 10 horas teóricas y 5 horas prácticas a la semana hasta completar 27 créditos por cada módulo en el semestre.

El objeto de estudio esgrime la problemática de este, en el contexto de los sujetos de estudio (estudiantes de 6to a 9no semestre de FCEAT) y del SEMOT que se aplica en las Licenciaturas en Negocios Internacionales, Negocios Agro tecnológicos y Contaduría Pública Fiscal.

Para dar sustento metodológico al desarrollo del módulo, el proceso de enseñanza aprendizaje del SEMOT se basa en las asignaturas durante los cuatro primeros semestres de la formación profesional en la FCEAT UAS. Estas asignaturas tienen como contenidos mínimos los tipos de investigación científica, niveles, enfoques, diseño, análisis de datos, estructura científica de documentos, agrupándose de la siguiente manera:

Tabla 2

SISTEMA DE CRÉDITOS DE LA OFERTA EDUCATIVA DE FCEAT 2017-2018

\begin{tabular}{|l|l|c|c|}
\hline Semestre & \multicolumn{1}{|c|}{ Asignatura o materia } & Horas & Créditos \\
\hline Primero & $\begin{array}{l}\text { Comprensión y producción de texto } \\
\text { científico }\end{array}$ & 54 horas-semestre & 4 \\
\hline Segundo & Metodología de la Investigación & 54 horas-semestre & 4 \\
\hline Cuarto & Métodos Cuantitativos y Cualitativos & 72 horas-semestre & 7 \\
\hline
\end{tabular}

Fuente: Elaboración propia con información de la Coordinación de Control Escolar de la Facultad de Ciencias Económicas, Administrativas y Tecnológicas (2017) de la Universidad Autónoma de Sinaloa. 
Estas asignaturas se correlacionan con asignaturas de introducción al conocimiento científico, creatividad e innovación, razonamiento y resolución de problemas, mientras que los objetivos del curso modular, hacen referencia al logro de los contenidos vinculados a la problemática de la metodología de la investigación, así como al desarrollo de conocimientos disciplinares y consolidación de habilidades y actitudes.

\section{RESULTADOS}

Se presentan solo los resultados obtenidos en relación con dos categorías (aprendizaje procedimental y aprendizaje actitudinal), enfocando esencialmente la identificación de desafíos y dificultades en el desarrollo del SEMOT, a partir del desempeño de los estudiantes y la percepción de sus actitudes, experiencias y sensaciones.

\section{CATEGORÍA DEL APRENDIZAJE REFLEXIVO, ACTIVO Y PROCEDIMENTAL}

En las gráficas 1 y 2, se puede observar que las dificultades metodológicas desarrolladas al implementar el SEMOT destacan que entre el 46,69\% y un 47,11\% de los estudiantes de las Licenciaturas en Negocios Internacionales, Negocios Agro tecnológicos y Contaduría Pública Fiscal de la FCEAT, tiene dificultades para comprender, asimilar y aplicar la metodología de la investigación.

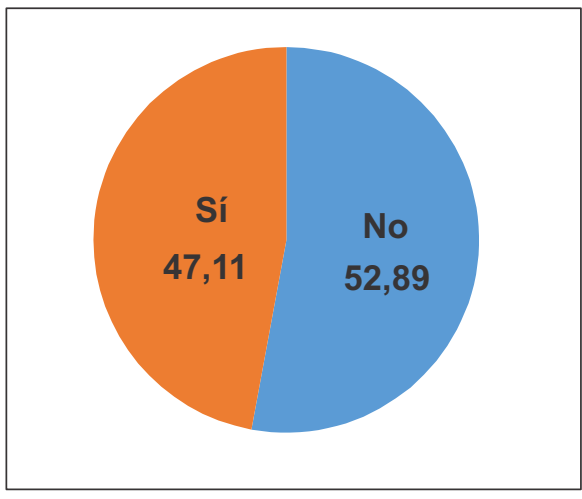

Gráfica 1. Dificultades en los conocimientos de metodología de la investigación. Fuente: Elaboración propia con información de cuestionario aplicado.

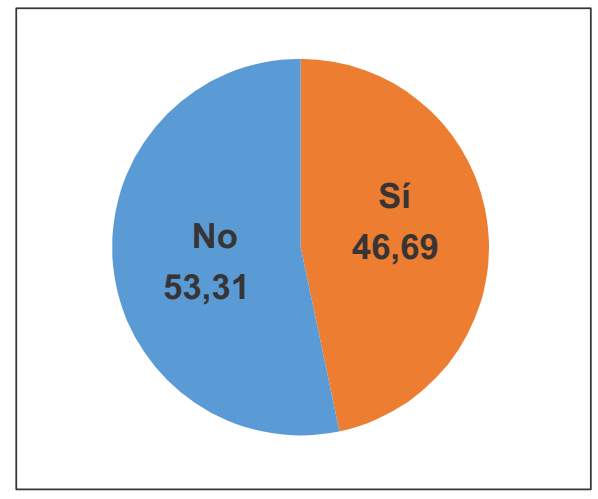

Gráfica 2. Apropiación del recuerdo en qué consisten las etapas del método científico. Fuente: Elaboración propia con información de cuestionario aplicado. 
Esto se explica, en función de que el 39\% de los estudiantes cursan el sexto semestre (tercer grado), que es cuando inicia la operación del SEMOT, lo que indica que las asignaturas relacionadas a la metodología de la investigación que previamente se cursaron, dejan a su paso un conocimiento metodológico endeble e inconsistente.

En las gráficas 3 y 4 se presenta que un $80 \%$ de los estudiantes aseguran no recordar los contenidos de las asignaturas previas al manejo del SEMOT, debido principalmente a las dificultades que se les presentaron en la comprensión de conceptos fundamentales vinculados a los temas de esas asignaturas previas, así lo revela el $57 \%$ de los estudiantes, dado que los conceptos no fueron aplicados; y por lo tanto, se dificultó el proceso de asimilación de este conocimiento.

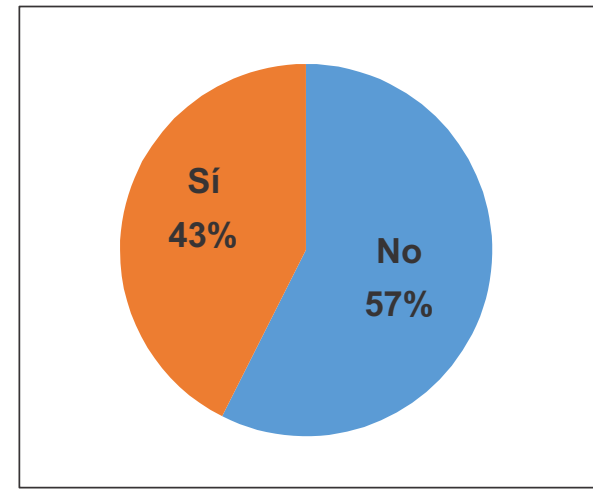

Gráfica 3. Dificultades en la comprensión de conceptos fundamentales vinculados a los temas de asignaturas previas.

Fuente: Elaboración propia con información de cuestionario aplicado.

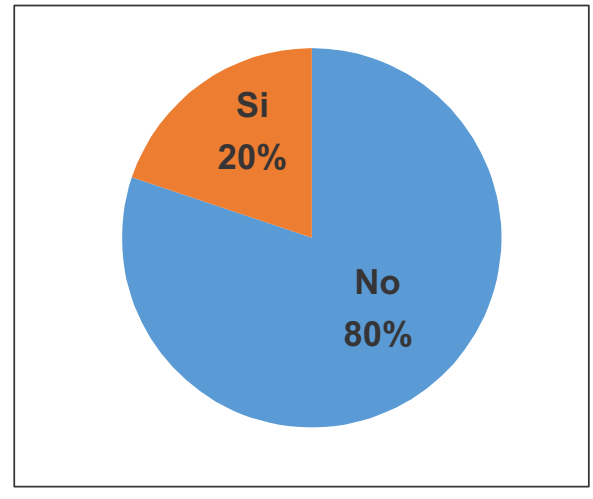

Gráfica 4. Se tienen presente los conocimientos aprendidos en cursos anteriores al SEMOT.

Fuente: Elaboración propia con información de cuestionario aplicado.

Al no aplicar los conocimientos aprendidos en curso anteriores al SEMOT, estos se han olvidado y al tenerlos presente, se limita su relación con la metodología de la investigación, lo que suscita dificultades en la comprensión y aplicación del SEMOT, provocando que los profesores del sexto semestre no podrían partir de conocimientos metodológicos previos y tendrán que retroalimentar y reeditar estos conocimientos para un mejor desempeño de los estudiantes en el SEMOT. 


\section{CATEGORÍA DEL APRENDIZAJE ACTITUDINAL Y PROCEDIMENTAL}

Respecto a esta categoría de análisis se preguntó a los 242 estudiantes que cursaban el SEMOT acerca de sus actitudes y sentires en el manejo y aplicación del conocimiento disciplinar y su vínculo con el proceso metodológico y la realidad del objeto de estudio.

Se encontró que un importante porcentaje del 73,55\% identifica los principios y valores bajo los cuales se desarrollaba su carrera, lo que permite generar en el estudiante la motivación para desarrollarse en el mercado laboral.

En la Tabla 3, se puede apreciar que la mayoría de los alumnos tienen una asimilación aceptable del pensamiento crítico $(57,44 \%)$, debido a que consideran que con este se generan soluciones a los problemas en las unidades de estudio, expresan el 63,64\% de los estudiantes encuestados. A diferencia de que la mayoría no logra asimilar el pensamiento lógico y tampoco encuentra su aplicabilidad en el campo de acción de su carrera, lo expresa el 52,07\% de los estudiantes.

Al desarrollar un pensamiento crítico, los estudiantes encuentran aplicabilidad de sus conocimientos; dado que los utilizan para resolver problemas relacionados con la disciplina que están cursando en el módulo que corresponda. En la

Tabla 3

ASIMILACIÓN DEL PENSAMIENTO CRÍTICO Y EL PENSAMIENTO LÓGICO

\begin{tabular}{|l|c|c|c|c|}
\hline \multirow{2}{*}{ Descriptor } & \multicolumn{2}{|c|}{$\begin{array}{c}\text { Respuesta Negativa } \\
\text { de Alumnos }\end{array}$} & \multicolumn{2}{c|}{$\begin{array}{c}\text { Respuesta Positiva } \\
\text { de Alumnos }\end{array}$} \\
\cline { 2 - 6 } & $\mathrm{N}$ & $\begin{array}{c}\text { Dato } \\
\text { Porcentual }\end{array}$ & $\mathrm{N}$ & $\begin{array}{c}\text { Dato } \\
\text { Porcentual }\end{array}$ \\
\hline $\begin{array}{l}\text { Conocimiento de los principios y valores } \\
\text { para desarrollar su profesión. }\end{array}$ & 64 & $26,45 \%$ & 64 & $73,55 \%$ \\
\hline Asimilación del pensamiento crítico. & 103 & $42,56 \%$ & 103 & $57,44 \%$ \\
\hline $\begin{array}{l}\text { Solución de los problemas utilizando el } \\
\text { pensamiento crítico. }\end{array}$ & 88 & $36,36 \%$ & 88 & $63,64 \%$ \\
\hline Asimilación del pensamiento lógico. & 126 & $52,07 \%$ & 126 & $47,93 \%$ \\
\hline
\end{tabular}

Fuente: Elaboración propia. 
gráfica 9 y 10, se aprecia que la mayoría de los estudiantes saben formular y gestionar proyectos de investigación (61\%), debido principalmente a que al estar cursando el SEMOT, el 58\% de los estudiantes, se han apropiado de los elementos que componen el planteamiento del problema.

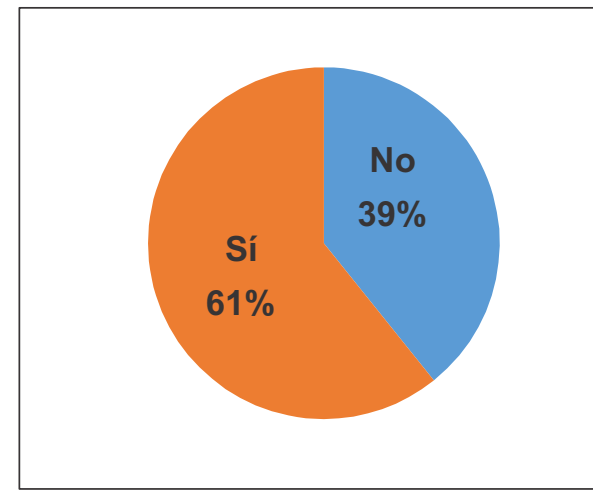

Gráfica 9. Formula y gestiona proyectos de investigación.

Fuente: Elaboración propia.

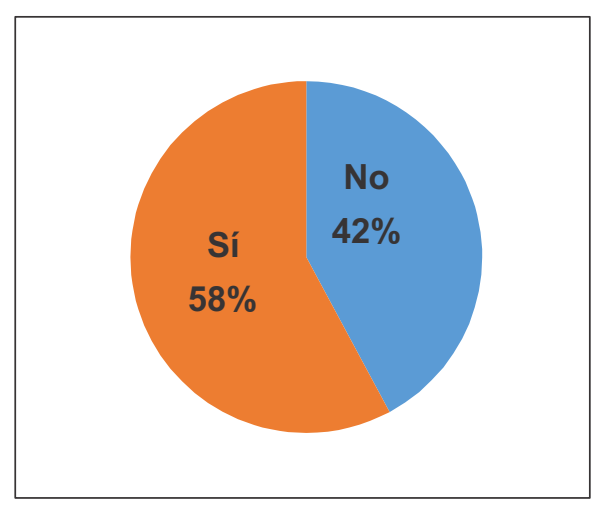

Gráfica 10. Desarrolla el planteamiento del problema.

Fuente: Elaboración propia.

Así también, esto se correlaciona con la gestión y búsqueda de información científica y empírica, encontrando que la mayoría de los estudiantes es más proclive a las gestiones de información publicitaria y científica, que a la búsqueda de información estadística. En la tabla 4, se puede identificar la comparación y el aspecto que les representa más dificultoso o desconocido a los estudiantes para registrar evidencia y contrastar sus hipótesis.

Se observa que mientras el $58,68 \%$ de los estudiantes saben gestionar información científica y un $56,61 \%$ información periodística seria e imparcial, un $77,27 \%$ de los estudiantes no saben o se reúsan a consultar y gestionar información estadística. Los que nos hace identificar una fragilidad del conocimiento en el procesamiento de datos estadísticos.

Otro aspecto que resultó interesante indagar, fue conocer los desafíos que los estudiantes tienen para explicar los términos técnicos de su investigación tales como robustez, validez, consistencia interna, varianza, fiabilidad, métodos, técnica y paradigma, que han sido estudiados en el curso de Metodología de la Investigación. 
Tabla 4

BÚSQUEDA Y GESTIÓN DE LA INFORMACIÓN CIENTÍFICA, PERIODÍSTICA Y ESTADÍSTICA

\begin{tabular}{|l|c|c|c|c|}
\hline \multirow{2}{*}{ Descriptor } & \multicolumn{2}{|c|}{$\begin{array}{c}\text { Respuesta Negativa } \\
\text { de Alumnos }\end{array}$} & $\begin{array}{c}\text { Respuesta Positiva } \\
\text { de Alumnos }\end{array}$ \\
\cline { 2 - 6 } & $\mathrm{N}$ & $\begin{array}{c}\text { Dato } \\
\text { Porcentual }\end{array}$ & $\mathrm{N}$ & $\begin{array}{c}\text { Dato } \\
\text { Porcentual }\end{array}$ \\
\hline Buscan información científica. & 100 & $41,32 \%$ & 142 & $58,68 \%$ \\
\hline $\begin{array}{l}\text { Buscar información periodística veraz } \\
\text { e imparcial. }\end{array}$ & 103 & $42,56 \%$ & 137 & $56,61 \%$ \\
\hline Saben buscar información estadística. & 187 & $77,27 \%$ & 55 & $22,73 \%$ \\
\hline
\end{tabular}

Fuente: Elaboración propia.

Ante la diferencia entre la gestión de información científica y periodística, respecto a la consulta de información estadística, podemos inferir que existe una diferencia en cuanto al uso de los métodos de investigación cualitativos y cuantitativos. Por lo que se procede analizar las dificultades y oportunidades que ofrece un enfoque y otro.

En las gráficas 11 y 12 , se puede observar que, aunque la mayoría de los estudiantes que desarrollan el SEMOT tienen conocimientos acerca de los paradigmas cuantitativo y cualitativo, los estudiantes son menos proclives a conocer y aplicar el método cuantitativo (71\%), mientras que son más los estudiantes que saben y aplican el método cualitativo (94\%). Esto indica que la mayoría de las investigaciones modulares que se producen en la FCEAT, son con un enfoque cualitativo y muy pocas sería combinando los dos enfoques.

Con base a estos datos, se puede inferir que la información empírica recogida refleja que existe provisión suficiente por parte de los estudiantes para abordar el aprendizaje desde el proceso metodológico del SEMOT, utilizando tanto el enfoque cualitativo con el cuantitativo. En tal sentido, se registra la evidencia que el SEMOT solventa el conocimiento disciplinar y metodológico.

Retomando el aspecto socioemocional, en la tabla 5, se pueden identificar algunos aspectos conductuales para realizar los trabajos de investigación a través del SEMOT. 


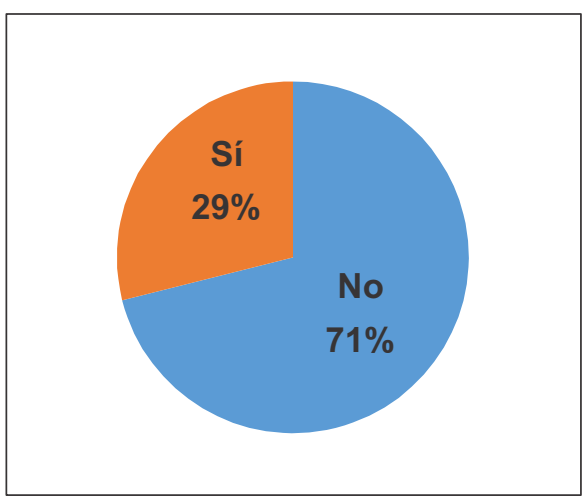

Gráfica 11. Sabe en qué consiste el desarrollo del método cuantitativo. Fuente: Elaboración propia.

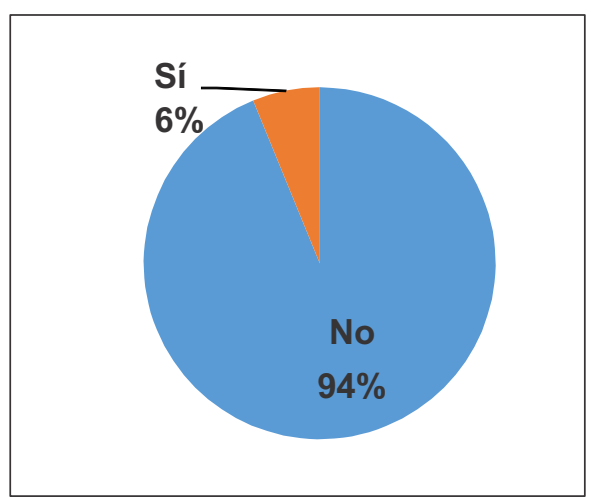

Gráfica 12. Sabe en qué consisten el desarrollo del método cualitativo. Fuente: Elaboración propia.

Tabla 5

ASPECTOS MOTIVACIONALES PARA REALIZAR INVESTIGACIÓN MODULAR

\begin{tabular}{|l|r|r|r|r|}
\hline \multirow{2}{*}{ Descriptor } & \multicolumn{2}{|c|}{$\begin{array}{c}\text { Respuesta Negativa } \\
\text { de Alumnos }\end{array}$} & \multicolumn{2}{c|}{$\begin{array}{c}\text { Respuesta Positiva } \\
\text { de Alumnos }\end{array}$} \\
\cline { 2 - 6 } & $\mathrm{N}$ & $\begin{array}{c}\text { Dato } \\
\text { Porcentual }\end{array}$ & $\mathrm{N}$ & $\begin{array}{c}\text { Dato } \\
\text { Porcentual }\end{array}$ \\
\hline $\begin{array}{l}\text { Les inspira la conducción científica de } \\
\text { sus profesores. }\end{array}$ & 157 & $64,88 \%$ & 85 & $35,12 \%$ \\
\hline $\begin{array}{l}\text { Sienten motivación para estudiar con } \\
\text { el SEMOT. }\end{array}$ & 45 & $18,60 \%$ & 197 & $81,40 \%$ \\
\hline $\begin{array}{l}\text { Les gusta realizar investigación modular. } \\
\text { Considera que es de utilidad y servicio }\end{array}$ & 18 & $7,44 \%$ & 224 & $92,56 \%$ \\
\hline la investigación modular. & 16 & $6,61 \%$ & 226 & $93,39 \%$ \\
\hline
\end{tabular}

Fuente: Elaboración propia. 
Se puede ver que pese a que a la mayoría de los estudiantes no les inspira la conducción científica y productividad académica de sus profesores $(64,88 \%)$, la mayoría consideran que el SEMOT es una estrategia productiva para su desempeño académico y profesional. La falta de inspiración investigativa desde la influencia de los profesores puede entenderse, dado que en el sistema modular solo son 3 de 9 profesores-investigadores de tiempo completo (33\%), los que se dedican a impartir docencia en el SEMOT, mientras que el 67\% de los investigadores están dedicados a actividades administrativas, estudiando o de año sabático.

Lo importante radica en la relevancia del SEMOT y la seriedad con la que los estudiantes lo desarrollan, dado que el 81,40\% de los estudiantes se sienten motivados al estudiar mediante el SEMOT, argumentando que este les permite ser proactivos, trabajar a sus tiempos y en vinculación con el sector productivo y social de la región; dado que lo que van conociendo disciplinalmente lo aplican en el lugar de una unidad de análisis (organización o empresa).

Con esto, se demuestra que un 92,56\% de los estudiantes les guste realizar investigación modular, porque consideran que es de utilidad y servicio a la socie$\operatorname{dad}(93,39 \%)$.

Para comprender una de las deficiencias en el aprendizaje de los estudiantes y los desafíos que impone el SEMOT, se encontró que las experiencias vividas en el SEMOT, les ofrece una oportunidad de desarrollo académico y profesional, partiendo del conocimiento de la unidad de análisis y la aplicación de los conocimientos adquiridos en el aula, bajo la participación de los estudiantes como actores de su propio aprendizaje.

Un aspecto que surge de las expresiones utilizadas por los estudiantes en la aplicación del cuestionario y las conversaciones que esto generó, fue la inspiración y la motivación con que enfrentaron el aprendizaje desde el SEMOT. Ellos expresan que la motivación es determinante para el desarrollo exitoso del SEMOT; sin embargo, prevalece la constante desilusión sobre algunos profesores que no se interesan o no saben desarrollar investigación a través del SEMOT, entendiendo que la conducción científica de la mayoría de los profesores que imparten el SEMOT, deja mucho que desear y esto obedece a que la mayoría de los profesores son maestros de asignatura y muy pocos son profesores investigadores dedicados al SEMOT (solo 3).

Expresan que antes de manejar el SEMOT, los estudiantes tenían una baja motivación personal, factor que afectó negativamente el desafío del aprendizaje; 
dado que, no se tenía la intención de comprender el proceso metodológico de manera activa para desarrollar el análisis y la reflexión. Sin embargo, al trabajar bajo el SEMOT, se despertó en la mayoría de los estudiantes el deseo por comprender mejor el proceso de investigación para aprender acerca de la disciplina y lo que se iba aplicar en el contexto socioeconómico de la región.

Consideran que sin motivación no aprenden, y como no aprenden, no producen trabajos de investigación modular. Se detecta que en la Licenciatura en Negocios Agro-tecnológicos se agudiza el problema de la simulación científica. Se supone que a consecuencia de la falta de profesores-investigadores que impartan los módulos, ya que, en su totalidad, son profesores de asignatura que no se dedican a hacer investigación. Esto será objeto de estudio en otra investigación diferente a esta.

Otras conjeturas vinculan la falta de influencia científica de los profesores a los estudiantes porque consideran que la mayoría de los profesores no hacen investigaciones serias y carecen de credibilidad y confianza científica. En muchos profesores, para la investigación no hay motivación interés personal, pues no le encuentran sentido ni le ven utilidad en su desarrollo docente.

También, algunos estudiantes reconocen el poco interés por el SEMOT, aludiendo que no corresponde a un conocimiento disciplinar y al no ser una materia específica de los negocios, la economía, la administración o la contaduría, le restan importancia, en tanto que otros estudiantes (la mayoría) hacen referencia a su importancia y a la necesidad de incentivar al alumno de algún modo para que el SEMOT les guste más.

Los estudiantes hacen una sugerencia a los docentes: Que apliquen el SEMOT de manera clara y proactiva; es decir, que de acuerdo vayan estudiando la disciplina del módulo, la vayan expresando desde un proceso de investigación, como una propuesta de enseñanza en la que se realicen actividades aplicables en el campo de actuación profesional para que sean interesantes y atractivas para los estudiantes.

Finalmente, con el SEMOT se eleva el interés personal; en virtud de que, con el proceso de investigación los estudiantes lograron entender mejor la disciplina porque aplicaron el conocimiento a problemas reales. El SEMOT al principio (en el sexto semestre) les resultaba difícil su comprensión y aplicación, lo que conducía a una resistencia declarada por parte de los estudiantes. De manera permanente los estudiantes hacían saber al profesor en turno que no harían la investigación; no 
obstante, de manera obligada tuvieron que desarrollarla, encontrándole mayor sentido y gusto del séptimo semestre en adelante. Esto demuestra que el SEMOT les resulto fácil conforme avanzaban en su desarrollo académico, por el tipo de razonamiento que observaban se estaba generando en ellos.

Reconocieron que hasta antes del sexto semestre, estaban acostumbrados a estudiar solo para los exámenes orales o escritos, pero del sexto semestre en adelante el SEMOT fue una expresión de aprendizaje a trasvés del proceso metodológico al que no estaban acostumbrados, pero dejan en claro que es la mejor estrategia para desarrollar un aprendizaje flexible, activo y procedimental que los orienta a resolver problemas de la realidad desde el conocimiento de una disciplina, convirtiendo un conocimiento inerte en un conocimiento dinámico que difícilmente se olvida, lo que alude a una mayor comprensión y reflexión desde una introspección reflexiva acerca de los contenidos disciplinares que serán recordados más fácilmente.

\section{CONCLUSIONES}

Los problemas de investigación en el campo de las ciencias sociales son multifacéticos y multifactoriales e inherentemente demasiado complejos para ser abordados por disciplinas individuales, de ahí el requerido desarrollo de la interdisciplina en las ciencias sociales.

En este estudio retoma la experiencia del SEMOT aplicado en FCEAT UAS. Bajo esta modalidad, a partir del sexto semestre, los estudiantes de tres licenciaturas del área de negocios internacionales, negocios agro-tecnológicos y contaduría pública fiscal, utilizan el esquema de investigación modular para cursar y acreditar uno los módulos que se cursan hasta el noveno semestre.

Esta experiencia, permite al estudiante introducirse en el mundo interdisciplinario de su objeto de estudio para aplicar su conocimiento en un caso real. Desde el inicio del curso modular, los alumnos se integran en equipos de investigación para facilitar el proceso de aprendizaje y colaboración interdisciplinaria para desarrollar las propuestas de intervención, implementar los aprendizajes en la práctica real y definiendo contextos y modelos de ejecución hasta finalizar la investigación modular, argumentando empírica, teórica y estadísticamente, respuestas a sus preguntas de investigación. Al final, el equipo de investigación modular hace la extensión de los hallazgos en un reporte de investigación científica con una estructura metodológica. 
En los equipos de alumnos en el SEMOT, se participa de diversas maneras (con visitas guiadas a la unidad física que se desea investigar, reuniones de grupos con los sujetos de estudio y encuentros con informantes clave para entrevistas u observaciones y la disertación en coloquios de investigación), enfrentando diversos desafíos y obstáculos, entre los que destaca la falta de apoyo de las organizaciones de la sociedad para dejar que los estudiantes de ciencias económicas y administrativas realicen una investigación aplicada.

Esto se puede explicar, por la carencia de la perspectiva investigativa e innovación que se tienen en las organizaciones de la sociedad, por la diversidad de intereses económicos, sociales y académicos entre las partes interactuantes, la complejidad que representa para los estudiantes el desarrollo de la metodología de la investigación y la resistencia a la gestión de proyectos de investigación; no obstante, a través de la participación colaborativa de los integrantes del equipo de investigación, se logra lidiar con los obstáculos para propiciar el aprendizaje desde investigaciones de gabinete que son también válidas.

Con el SEMOT se desarrolla la investigación interdisciplinaria, ampliando el proceso de aprendizaje y los hallazgos, pero, sobre todo, abriendo nuevas líneas de investigación con alto sentido de responsabilidad social. La participación de los estudiantes en el SEMOT se correlaciona positivamente con resultados beneficiosos en el proceso de aprendizaje a través de la interdisciplina.

Se destaca, que pese a que no se conocen los niveles de ventaja que permite la interdisciplina, observamos resultados positivos con el SEMOT en cinco categorías de impacto: 1 . avances en el descubrimiento científico, 2. comprensión del basamento teórico en relación con los hechos reales, 3. se amplía la participación de los estudiantes investigadores, 4. mejora la productividad académica y 5. Mejora la disertación y el discurso interdisciplinario.

Con el SEMOT y el enfoque interdisciplinario, se podría generar una mayor consolidación en la producción académica y científica, en beneficio de la sociedad y las organizaciones que operan desde las ciencias económicas y administrativas.

Con este estudio, queda evidencia para considerar que la participación de los estudiantes en el SEMOT impartido en FCEAT UAS, es un método con un enfoque interdisciplinario que atiende cinco criterios originales del aprendizaje investigativo.

Desde la experiencia de los estudiantes, queda documentado que respecto a la asimilación y retención del conocimiento disciplinar, es más fácil recordar lo 
que se aprendió en la aplicación del SEMOT. El hecho de estudiar un conjunto de contenidos disciplinares solamente para un examen, no significa la asimilación y el reforzamiento del conocimiento, dado que no se aplica en un contexto real y mediante el proceso de investigación, mientras que cuando se hace aplicándolo, adquiriendo relevancia si los estudiantes pueden recordar el contexto en que se aplicó y las condiciones bajo las cuales se generó, logrando que el conocimiento queda firme y se recuerde.

En esta idea, la aplicación activa del conocimiento permite transferir el conocimiento. Trasladar el conocimiento implica aprender una disciplina en una situación y un contexto real, retroalimentándose de otras disciplinas. Esto en esencia es el SEMOT, un conjunto de actividades en las que confluyes varias disciplinas para generar un conocimiento estructurado mediante el proceso de investigación científica para resolver un problema de la realidad, generando la reflexión, la práctica, el análisis, la retroalimentación, el servicio y la extensión del conocimiento que fue adquirido, asimilado y aplicado.

Por todo esto, la decisión de continuar defendiendo el SEMOT en la educación superior y más específicamente en las Licenciaturas que se imparten en FCEAT UAS, implica un fuerte compromiso con la enseñanza, la aplicación y el seguimiento socialmente responsables de la metodología de la investigación, lo que significa seguir debatiendo y reflexionando acerca de las ventajas y oportunidades que tiene el sistema de enseñanza que caracteriza nuestra Facultad, pero sobre todo, continuar produciendo conocimiento científico.

Se sugiere que se genere y desarrolle la aplicación de una sexta categoría de análisis para afianzar aún más el SEMOT, esta correspondería a una mayor vinculación con el sector productivo interesado en desarrollar investigación científica en proyectos, productos y procesos. Lo que permitiría un mayor desarrollo de la comunidad de investigación, avanzar en la exploración, descripción y descubrimiento de hechos, contrastación de hipótesis y comprensión científica para difundir los resultados en beneficio de la sociedad.

Además, para fomentar una actitud positiva hacia la investigación científica entre los estudiantes de Licenciatura, se deben proporcionar becas que ayuden a financiar sus proyectos de investigación. Esto permitirá fomentar una mayor participación en las actividades científicas para aprovechar las oportunidades de ingresar a un posgrado de calidad en el que se pueda mantener una beca durante sus años de formación científica. 
Con esta investigación, se focaliza en forma detallada la caracterización y comprensión de las dificultades teóricas, conceptuales y metodológicas que poseen los estudiantes al ingresar al sexto semestre y al aplicar el SEMOT. Se destaca que en el desarrollo del SEMOT se ven reflejados y generados conceptos como: aprendizaje activo, aprendizaje profundo; transferencia de aprendizaje, aprendizaje reflexivo, aprendizaje por imitación, andamiaje metodológico para el aprendizaje, entre otros. Todos estos con un alto sentido de idoneidad e inocuidad para el SEMOT.

Por último, las dificultades teóricas, conceptuales y metodológicas que enfrentan los estudiantes, no deben atribuirse únicamente al estudiante y su aprendizaje, sino que deben comprenderse desde la enseñanza y la práctica docente, a la luz de la aplicación del SEMOT. Se descubrió que la motivación personal es fundamental para el mejor desarrollo del proceso de investigación y la mayor comprensión disciplinar de los contenidos y los aprendizajes; por lo que, será necesario trabajar y aplicar estrategias socioemocionales, motivacionales, inspiracionales y aspiracionales que coadyuven al aprendizaje tanto actitudinal como al procedimental.

Resulta interesante revelar los desafíos, dificultades, ventajas y oportunidades que el SEMOT ofrece por cada una de las Licenciaturas; por lo que, queda en reserva para un siguiente estudio en que se desagreguen los datos para identificar en cuál de las Licenciaturas tiene un mayor impacto el SEMOT, además de identificar cuáles serían los determinantes de ello.

\section{REFERENCIAS BIBLIOGRÁFICAS}

Abramo, G., D'Angelo, C.A., and Di Costa, F. (2018). Specialization versus diversification in research activities: the extent, intensity and relatedness of field diversification by individual scientists, Scientometrics, 112 (3), p.p. 1403-1418, DOI: 10.1007/s11192-017-2426-7.

Aiello-Sindoni, M. (2009). Dificultades en el aprendizaje de la metodología de la investigación, magis, Revista Internacional de Investigación en Educación, 2 (3), p.p.141-156.

Aranda, C., Luna, L., Costanzo, N., Contissa, V., Gámez, N., Godoy, P., and Zuccala, K. (2014). Conservación e investigación en el área de Antropología Biológica del Museo Etnográfico J. B. Ambrosetti (Facultad de Filosofía y Letras, Universidad de Buenos Aires, Argentina), Antropologia Portuguesa, 30, p.p. 11-28, DOI: 10.14195/2182-7982_31_1.

Auerbach, A.J.J., and Andrews, T.C. (2018). Pedagogical knowledge for active-learning instruction in large undergraduate biology courses: a large-scale qualitative investigation of instructor thinking, International Journal of STEM Education, 5 (1), art. no. 19, p.p.1-25, DOI: 10.1186/s40594-018-0112-9. 
Belardo, C., Burrows, A.C., and Dambekalns, L. (2017). Partnering science and art: Preservice teachers' experiences for use in pre-collegiate classrooms, Problems of Education in the 21st Century, 75 (3), p.p. 215-234.

Bibri, S.E. (2018). A foundational framework for smart sustainable city development: Theoretical, disciplinary, and discursive dimensions and their synergies, Sustainable Cities and Society, 38, pp. 758-794, DOI: 10.1016/j.scs.2017.12.032.

Cannizzaro, S. (2013). Where Did Information Go? Reflections on the Logical Status of Information in a Cybernetic and Semiotic Perspective, Biosemiotics, 6 (1), p.p. 105-123, DOI: $10.1007 / \mathrm{s} 12304-012-9154-4$.

Chen, W. (2018). Introduction to Research: A New Course for First-Year Undergraduate Students, Journal of Chemical Education, 95 (9), p.p. 1526-1532, DOI: 10.1021/acs.jchemed. 8 b00102.

Dem, E.S., Rodríguez-Labajos, B., Wiemers, M., Ott, J., Hirneisen, N., Bustamante, J.V., Bustamante, and M., Settele, J. (2018). Understanding the relationship between volunteers' motivations and learning outcomes of Citizen Science in rice ecosystems in the Northern Philippines, Paddy and Water Environment, 16 (4), p.p. 725-735, DOI: 10.1007/s10333. 018-0664-9.

Dorfner, T., Förtsch, C., Germ, M., and Neuhaus, B.J. (2018). Biology instruction using a generic framework of scientific reasoning and argumentation, Teaching and Teacher Education, 75, p.p. 232-243, DOI: $10.1016 /$ j.tate.2018.07.003.

Durham, M.F., Knight, J.K., Bremers, E.K., DeFreece, J.D., Paine, A.R., Couch, B.A. (2018). Student, instructor, and observer agreement regarding frequencies of scientific teaching practices using the Measurement Instrument for Scientific Teaching-Observable (MISTO), International Journal of STEM Education, 5 (1), art. no.31, DOI: 10.1186/s40594-018-0128-1.

Facultad de Ciencias Económicas, Administrativas y Tecnológicas [FCEAT (2017)]. Datos de matrícula escolar cohorte 2017-2018, México, Universidad Autónoma de Sinaloa.

Frank, A.M., Froese, R., Hof, B.C., Scheffold, M.I.E., Schreyer, F., Zeller, M., and Rödder, S. (2017). Riding alone on the elevator: A class experiment in interdisciplinary education, Learning and Teaching, 10 (3), p.p. 1-19, DOI: 10.3167/latiss.2017.100302.

Guevara Saucedo (1982). El Sistema Modular de las Escuelas y Facultades de Medicina Veterinaria yZootecnia, UAM-X, México.

Huppenkothen, D., Arendt, A., Hogg, D.W., Ram, K., VanderPlas, J.T., and Rokem, A. (2018). Hack weeks as a model for data science education and collaboration, Proceedings of the National Academy of Sciences of the United States of America, 115 (36), p.p. 8872-8877, DOI: $10.1073 /$ pnas.1717196115.

Kimmons, R. and Veletsianos, G. (2018). Public Internet Data Mining Methods in Instructional Design, Educational Technology, and Online Learning Research, TechTrends, 62 (5), p.p. 492-500, DOI: $10.1007 / \mathrm{s} 11528-018-0307-4$.

Koohi-Var, T. and Zahedi, M. (2018). Cross-domain graph based similarity measurement of workflows, Journal of Big Data, 5 (1), art. no. 18, p.p. 1-16, DOI: 10.1186/s40537-018-0127-6. 
Kruit, P.M., Oostdam, R.J., van den Berg, E., and Schuitema, J.A. (2018). Assessing students' ability in performing scientific inquiry: instruments for measuring science skills in primary education, Research in Science and Technological Education, 36 (4), p.p. 413-439, DOI: 10.1080/02635143.2017.1421530.

Liu, Z., Kettimuthu, R., Foster, I., and Beckman, P.H. (2018). Toward a smart data transfer node, Future Generation Computer Systems, 89, p.p. 10-18, DOI: 10.1016/j.future.2018.06.033.

Luckie, D., Sweeder, R., and Bellon, R. (2013). Bringing relationships alive through interdisciplinary discourse (BRAID), International Journal of Pedagogy and Curriculum, 19 (3), p.p. 133-144, DOI: 10.18848/2327-7963/CGP/v19i03/48920.

Martinez, G., Armaroli, C., Costas, S., Harley, M.D., and Paolisso, M. (2018). Experiences and results from interdisciplinary collaboration: Utilizing qualitative information to formulate disaster risk reduction measures for coastal regions, Coastal Engineering, 134, pp. 62-72, DOI: 10.1016/j.coastaleng.2017.09.010.

MacLeod, M. (2018). What makes interdisciplinarity difficult? Some consequences of domain specificity in interdisciplinary practice, Synthese, 195 (2), p.p. 697-720, DOI: 10.1007/s11229-016-1236-4.

Piso, Z., O'Rourke, M., and Weathers, K.C. (2016). Out of the fog: Catalyzing integrative capacity in interdisciplinary research, Studies in History and Philosophy of Science Part A, 56, p.p. 84-94, DOI: 10.1016/j.shpsa.2016.01.002.

Ruuskanen, T., Vehkamäki, H., Riuttanen, L., and Lauri, A. (2018). An exploratory study of the learning of transferable skills in a research-oriented intensive course in atmospheric sciences, Sustainability (Switzerland), 10 (5), p.p. 1-20, DOI: 10.3390/su10051385.

Stark, E., Kintz, S., Pestorious, C., and Teriba, A. (2018). Assessment for learning: using programmatic assessment requirements as an opportunity to develop information literacy and data skills in undergraduate students, Assessment and Evaluation in Higher Education, 43 (7), p.p. 1061-1068, DOI: 10.1080/02602938.2018.1432029.

Stubbs, E.A., Zimmerman, A.R., Warner, L.A., and Myers, B.E. (2018). Reflecting on a multidisciplinary collaboration to design a general education climate change course, Journal of Environmental Studies and Sciences, 8 (1), p.p. 32-38, DOI: 10.1007/s13412-017-0451-8.

Sullivan, S., and Puntambekar, S. (2019). Learning with multiple online texts as part of scientific inquiry in the classroom, Computers and Education, 128, p.p. 36-51, DOI: 10.1016/j.compedu.2018.09.004.

Ünver, S., Semerci, R., Özkan, Z.K., and Avcibaşi, I. (2018). Attitude of Nursing Students Toward Scientific Research: A Cross-Sectional Study in Turkey, Journal of Nursing Research, 26 (5), p.p. 356-361, DOI: 10.1097/JNR.0000000000000244

Vezzani, C., Vettori, G., and Pinto, G. (2018). University students' conceptions of learning across multiple domains, European Journal of Psychology of Education, 33 (4), p.p. 665-684, DOI: 10.1007/s10212-017-0349-6.

Wikström, E., Eriksson, E., Karamehmedovic, L., and Liff, R. (2018). Knowledge retention and age management - senior employees' experiences in a Swedish multinational company, Journal of Knowledge Management, 22 (7), p.p. 1510-1526, DOI: 10.1108/JKM-09-2017-0442. 